\title{
Research on the Influence of Micro Blog Development on College Students and the Countermeasures
}

\author{
Limei Zhao ${ }^{1}$, Qiang Liu ${ }^{1}$ and Enyang Gao ${ }^{2^{*}}$ \\ ${ }^{1}$ Jilin Engineering Normal University .Changchun; China; \\ ${ }^{2}$ Shenyang Jianzhu University, Shenyang, China \\ Yangnx1980@163.com \\ ${ }^{*}$ The corresponding author
}

Keywords: Micro-blog; Social media; College students; Information transmission; Ideological and political education

\begin{abstract}
In recent years, micro-blog has become an important part of College Students' life, and become a new way to the innovation of College Students' education. The rapid development of micro Bo cause the attention of scholars, but microblogging on College Students' study on the influence of vision is relatively narrow, research of high repetition rate, mainly concentrated in Ideological and political education of College Students' readers in this field through the microblogging. Micro-blog has hindered the development of College Students' cognitive ability and non-critical ability. Long term use of micro-blog will lead to college students addicted to micro-blog. On these issues, through the collection of prizes, regulate the network environment, the popularity of real name system to use and limit the use of time to solve. Through the collation and discussion of the relevant literature, this paper discusses the shortcomings of the current research and the direction of future research.
\end{abstract}

\section{An Overview of the Reasons why College Students Use Micro-blog}

Exploring the reason why the university students use micro-blog is an important premise to study the influence of micro-blog on college students, and it is also one of the problems that the researchers are more concerned about. Most of the existing researches focus on the reasons why college students use micro-blog and micro-blog's attention. With the rapid development of micro-blog, micro-blog has become a college student information circle, circle of public opinion, showing a rapid development of public opinion, the spread of a wide range of public opinion changes and other characteristics.

Scholars Have Explored the Deep Reasons for the Close Combination of Micro-Blog and College Students. The scholars have summarized the unique characteristics of micro-blog, the instant of information dissemination, and the grassroots of the use of information, interactive and personalized display. In addition, the micro blog as a kind of open emerging network of communication tools has attracted more and more users, especially the young college students are generally favored. Among them, 90 students have become micro blog users of the main force. Micro blog in changing the public media habits and the mode of transmission of information, the user can enjoy the 4A level (i.e., anytime, anywhere, anyone, anything) services. At the same time, it also plays a dissemination of ideas and the construction of the concept of value oriented function, the set of network media social function in a body. From a certain point of view, micro blog has opened up a new space for the university education, and provides a new method and means. Blog makes the original relatively small education space into a whole society, open education space for the whole society to create a good network system environment and education model.

However, "freedom, openness, sharing," micro blog spirit, this spirit and easy to make students thinking irrational, resulting in their wayward character, also affect the college students' concept of rational set up, easy to cause the students "excessive liberalization, law discipline consciousness weak, luring college students on the behavior and consciousness of a qualitative change, on college students form correct world outlook, values, life have a negative impact on, which also makes the dominant 
position of the school education by the unprecedented impact. As a higher education worker, a clear understanding of the micro blog on the impact of college students after 90 is worthy of our attention and research.

In summary, combining micro blog and college students can be summarized as: first, microblogging for college students to provide a platform for self-display; second, microblogging has expanded the students' communicative range; the third microblogging to enable students to obtain the needed information of.

The Social Characteristics of College Students themselves Is an Important Reason Why It Is Combined with Micro-Blog. Most college students are in the middle of youth (ages 18-24) and physical development of the individual is nearing completion, psychological development gradually mature, college students because of the characteristics of its environment, and showing a characteristic is as follows: self-awareness; improve the degree of social; a feeling of loneliness. So, college students with its unique identity and personality characteristics, become one of the main use of the group of micro-blog.

In modern society, everyone in the search for a "personalized", because the character is a symbol of innovation, marking the self-confidence, embodies the tension, and college students through the use of microblogging can reduction "personality" and to satisfy their own sense of social existence. Visible, the existence of multiple values to promote the emotional development of college students have a certain social dependence, college students can express their inner feelings with micro-blog, the release of multiple pressures in life. Therefore, the urgent need for college students to seek self-development and psychological appeal is one of the reasons why the use of micro-blog. In a word, the special objective existence of the College Students Group determines its unique psychological and social characteristics.

Emerging media micro-blog's emergence just to achieve this function. This has become the real basis of the combination of the two.

In the study of college students pay attention to the micro Bo content, most of the literature that college students pay attention to the micro Bo content and students use microblogging unity, in content with concentration, radiation and other characteristics.

First, "focus on self" as the main content. Students micro Bo main content is: their opinions or emotional catharsis, show interest in hobbies or released just anecdotes, which occupy more than 50\% of the respondents. Micro-blog to avoid the reality of face to face interpersonal embarrassment, through the micro-blog this virtual media, college students can fully express their feelings, cultivate good interests and hobbies, to achieve the perfect individual. However, this kind of way to cut off the reality of the exchange also has the inevitable negative effect. So they once on this platform suffered setbacks in communication, it is easy to breed paranoia, fear, sensitive and numb, indifferent etc. psychological characteristics, even to the individual psychology. Secondly, college students can realize the social concern by micro-blog. In the absence of special events, college students pay more attention to the general entertainment topic, after special events occur mainly around special events. For example, the two sessions, as well as in 2012 the major anti-corruption events around the issue of corruption in the sun to discuss the sun against micro-blog. But now, there are some people accused of corruption, there are some important defects, such as the use of Internet users more

"Official is corrupt" presumption of guilt, microblogging has become the virtual space of the guillotine, Robespierre revolutionary carnival; public opinion trial, legal back; if the defendant in the micro Bo no retort, right relief lack and so on.

\section{Research on the Impact of Micro-blog on College Students}

Influence of micro blog on college students, scholars views are basically the same, that microblogging all aspects of life on college students have caused varying degrees of impact, especially in college students' psychological, value view, interpersonal and ideological and political education and so on. Of course, this effect is dialectical, including two aspects of positive factors and negative factors. 
Micro-Blog Has a Certain Degree of Impact on the Psychology of College Students. Due to the rapid pace and pressure of a variety of new media, learning, life, and so on, college students have less direct contact with people. Through the micro Bo, they can talk out their inability to express feelings, life, and get the "fans" enlighten, communication and understanding. This kind of network communication will help to form an equal discussion atmosphere, without being bound by identity, status and so on, so that they can get self-satisfaction in mind. But in micro blog, everyone can be a maker of information and communication, the lack of real life in the correct guidance of checking and, will inevitably cause adverse information pollution and flooding have a great impact to the development of College Students' mental health. In addition, college students are addicted to the virtual world easily lead to mental illness, microblogging for college students provides a new media experience, at the same time hidden worries about the excessive use of Internet addiction. Micro-blog provides the exchange experience to enable students to obtain a great spiritual satisfaction, the whole business of this virtual circle, and the real world of the struggle to relax.

Micro-Blog's Impact on College Students' Values Can not Be Ignored. From the previous research literature, it is a negative impact. The blog culture has opened up a new space for the youth generation of information, provides a new method and means for the whole society to communicate and exchange to create a good environment. But the blog culture also cater to the many ideological changes in young people, induced young generation changes occurred in the matter of behavior and awareness and conception of the younger generation a correct world outlook, outlook on life, have a negative impact, resulting in a flood of young generation "excessive liberalization, the rule of law and discipline consciousness weak" trend of thought. Micro-blog to make the mainstream values of the traditional way to guide the impact, easily lead to the deviation of the value of cognition, at the same time, the campus culture of college students to a certain extent, the positive guidance is weakened. Multiple led directly to the values in college students in the emotional needs of pluralism, the absolute worship of authority, absolute single emotion need is gone, replaced by multiple colorful emotional needs, and the formation of complex emotional need structure. Therefore, in the future research must attention and study of micro Bo communication functions, actively guide students correctly use microblogging; pay attention to the education of network environment, build a healthy and harmonious network environment for users of the microblogging, help students establish the correct network (micro Bo) concept.

\section{On the Extension of Micro-Blog's Influence on College Students}

Micro-blog provides a low threshold, low cost, high efficiency, social communication form, for the realization of the information age of interpersonal communication made a significant contribution. Cao Lin pointed out that microblogging is a society, the society has what problem, were projected to the micro Bo, because microblogging is geometrically the propagation speed and breadth, so it can be social problems magnified. He also summed up the ten characteristics of the micro blog, including strong crowd atmosphere and spread of scripts, bad good news, is vulnerable to manipulation, elite, closed, entertainment, lack of originality, the expression of fragmentation and emotional. Although these features are not unique to micro-blog, but micro-blog in which to act as an amplifier function, these features are infinitely magnified. Micro-blog's rapid rise to the life of college students has brought changes, how to effectively apply micro-blog in the daily life of college students has become an important task for ideological and political education in Colleges and universities and college students. And at present, the domestic research on the impact of micro-blog on college students is strong; the research field of vision is not very broad. Overall, the study did not form a complete theoretical system. Most research from the perspective of Ideological and political education in Colleges and universities, the repetition rate is high; there is no in-depth, detailed discussion. At the same time, basic research is also the framework of a unified, more limited thinking. The author thinks that in the course of study must make clear micro-blog. The characteristics of this new media, mining micro-blog and college students in combination with the inherent consistency of the depth of the impact 
of micro-blog on College students. The famous American media culture study and critic Neil Postman once pointed out: "destroy us are not what we hate, but rather what we love." Micro-blog is also so, if we cannot reasonably and effectively use the advantages of micro-blog, then we will eventually become its slaves, losing us as a person's social mentality and subjective initiative. Therefore, the spread function of micro-blog research is particularly important.

\section{References}

[1] Zhu Danhui. Study on the phenomenon and its guidance of college student's blog [D]. Henan University of Science and Technology, 2011

[2] Zeng Zeng. The function and application of blog in the ideological and political education of college students [D]. Huazhong Normal University, 2012

[3] Zheng Zhibin, Zhao Yingyan. Investigation and analysis of the use of college student's blog [J]. Contemporary spread, 2007, 03:67-70.

[4] Shi Shi, Zhang Zhijie. Investigation on the status quo of blog usage of college students [J]. Journal of Southwestern Normal University (NATURAL SCIENCE), 2007, 03:172-176. (in Chinese).

[5] Xia Zuxin. A discussion on the negative impact of blog communication on college students [J]. Science and technology information, 2011, 13:172-173.

[6] Zhang Minxia. Micro blog and its application in the library [J]. Library science research, 2010, $10: 55-58+77$.

[7] Yuan Nayu. Value analysis of micro blog in library application [J]. Books and information, 2010, 03:104-106. 